\title{
Evaluación del efecto de fertilización, aplicación de estiércol y absorción de elementos en el rendimiento de la secuencia papa-kiwicha, evaluado mediante la técnica del elemento faltante
}

\author{
Evaluation the effect of fertilization, application of manure \\ and nutrient uptake in the yield of the sequence potato-kiwicha, \\ evaluated using the omitted element technique.
}

\author{
Edgardo Alaluna G. ${ }^{1}$ y Sven Villagarcía $\mathrm{H} .^{2}$
}

\section{RESUMEN}

El presente trabajo evalúa el efecto de la fertilización al suelo, incorporación de estiércol y fertilización foliar de los elementos $\mathrm{N}, \mathrm{P}, \mathrm{K}, \mathrm{Mg}, \mathrm{Fe}, \mathrm{Mn}$ y $\mathrm{Zn}$, los cuales fueron evaluados mediante la técnica del elemento faltante. El experimento se condujo en un suelo de textura tipo arena de la Costa central del Perú y se usaron como plantas indicadoras los cultivos de papa y kiwicha, este último para evaluar el efecto residual de cada uno de los tratamientos. La fertilización al suelo para cada elemento en mg $\mathrm{kg}^{-1}$ fue de $200=\mathrm{N}, \mathrm{P}_{2} \mathrm{O}_{5}$ y $\mathrm{K}_{2} \mathrm{O} ; 50=\mathrm{MgO}$ y Fe; $25=\mathrm{Mn}$ y $\mathrm{Zn}$. Se utilizó el diseño completo al azar con arreglo factorial, con tres repeticiones y conducido en macetas de $4 \mathrm{~kg}$ de suelo. La evaluación consistió en medir el rendimiento de materia seca total y absorción total de nutrientes analizados en los tejidos de la papa y kiwicha. Con la incorporación de 25 t/ha estiércol es posible suplir los requerimientos nutricionales de los elementos $\mathrm{Fe}, \mathrm{Mn}$ y $\mathrm{Zn}$ para el cultivo de la papa; así mismo, mediante este procedimiento se puedo suplir parcialmente los requerimientos de $\mathrm{K}$ y $\mathrm{Mg}$ y de manera muy limitada los de $P$ y $N$. En la kiwicha el tratamiento completo mostró ligera depresión en su rendimiento comparado con las ausencias de $\mathrm{Mg}, \mathrm{K}, \mathrm{Mn}$ y $\mathrm{P}$, pues estos elementos fueron favorecidos por la mineralización del estiércol adicionado al cultivo anterior. Asi mismo, el efecto residual del estiércol favoreció la disponibilidad del N, P y K, los cuales incrementaron la producción en 1025\%, $42 \%$ y $13 \%$ con relación a los tratamientos sin estiércol, y mostró efecto negativo frente a las ausencias de $\mathrm{Mn}$, Fe y $\mathrm{Zn}$ debido a la quelación de estos con la materia orgánica. La fertilización foliar sólo mostró efecto positivo en los tratamientos con buen balance nutricional a nivel del suelo como en los casos del completo, testigo y en ausencia de elementos menores. En los tratamientos con estiércol se observó efecto positivo significativo en la absorción de macro y microelementos en la papa; en la kiwicha no fue notorio y sólo contribuyó a la absorción del fósforo de manera significativa. La fertilización foliar únicamente favoreció la absorción de los elementos en los tratamientos con un balance adecuado de nutrientes a nivel del suelo.

Palabras clave: fertilización, suelo arenoso, kiwicha

\section{ABSTRACT}

This trial was established to study the effects of foliar fertilization, incorporation of manure and soil application of $\mathrm{N}, \mathrm{P}, \mathrm{K}, \mathrm{Mg}, \mathrm{Fe}, \mathrm{Mn}$ and $\mathrm{Zn}$; these nutrients were evaluated through the missing element technique. The trial was conducted in a sandy soil from central coast of Peru. Potato and kiwicha were used as test plants, the latter being used to evaluate the residual effect in each treatment. Soil fertilization for each element in mg. $\mathrm{kg}^{-1} / \mathrm{pot}$ were $200=\mathrm{N}, \mathrm{P}_{2} \mathrm{O}_{5}$ and $\mathrm{K}_{2} \mathrm{O} ; 50=\mathrm{MgO}$ and $\mathrm{Fe}$ and $25=\mathrm{Mn}$ and $\mathrm{Zn}$. A complete randomized design with factorial arrangement and three replications was used. Replications were $4 \mathrm{~kg}$ soil pots. The evaluation consisted in measuring the total dry matter yield and total nutrient uptake in the potato-kiwicha. Applying $25 \mathrm{tn} / \mathrm{ha}$ of manure makes possible to supply the nutritional requirements of $\mathrm{Fe}, \mathrm{Mn}$ and $\mathrm{Zn}$ for potato crop. It also partially supplies the requirements of $\mathrm{K}$ and $\mathrm{Mg}$ and in a very limited extention, $\mathrm{P}$ and $\mathrm{N}$ requirements. In kiwicha crop, the complete treatment

1 Ing. Agrónomo. Universidad Nacional Agraria la Molina. E-mail: alaluna@ lamolina edu pe

2 Docente Principal del Departamento de Suelos y Fertilizantes. Universidad Nacional Agraria La Molina. Departamento de Suelos y fertilizantes Av. La Universidad s/n ta Molina. T. $3495669-210$. 
showed a slight yield deppresion as compared with omitted $\mathrm{Mg}, \mathrm{K}, \mathrm{Mn}$ and $\mathrm{P}$ as these elements were enhanced by last year manure mineralization. Manure applications significatively enhanced the N, Mn, $\mathrm{P}, \mathrm{K}, \mathrm{Zn}$ and $\mathrm{Fe}$ availability, which increased the total dry matter yield in kiwicha by 1025,42 and $13 \%$ as compared with no manure treatments, and showed a deppresive effect compared with omitted $\mathrm{Mn}, \mathrm{Zn}$ and $\mathrm{Fe}$, due to the complexing of these elements with organic matter. Foliar fertilization showed a positive effect only in treatments with adequate soil nutritional imbalance as in complete treatment, test and omitted microelements treatments. A significatively positive effect was observed in manure treatments in macro and microelement uptake in potato. In kiwicha, this effect was not clear and only enhanced significatively the phosphorus uptake. Foliar fertilization enhanced the uptake only in treatments with an adequate soil nutrient in balance.

Key words: fertilization, sandy soil, kiwicha

\section{INTRODUCCIÓN}

En la costa peruana especialmente en la zona Central se practica la agricultura moderna, intensiva y de alta rentabilidad en suelos aluviales y de irrigación. Estos últimos son de textura gruesa y sin estructura, a diferencia de los suelos de valle. Asimismo estos suelos se caracterizan por tener bajos contenidos de nitrógeno y fósforo con un contenido medio de potasio; en estos suelos se están conduciendo plantaciones muy costosas de hortalizas, frutales, etc., dirigidos principalmente al mercado externo en los cuales no sólo se están realizando aplicaciones masivas de estiércoles y de N, P y K, sino también de $\mathrm{Mg}, \mathrm{Fe}, \mathrm{Mn}$ y $\mathrm{Zn}$ sin tener la seguridad de que si hace falta $o$ no.

\section{Generalidades}

A través de los análisis químicos del suelo y de la producción de materia seca de las plantas que crecen en ellos, es posible la calibración de técnicas químico-analíticas y la estimación del estado nutricional de un suelo. En estos estudios, es común el uso de correlaciones entre respuestas de los cultivos a la aplicación de nutrientes y la cantidad de nutrientes detectados en los tejidos por técnicas analíticas (Tisdale y Nelson, 1970).

\section{EVALUACIÓNDE LA FERTILIDAD DELSUELO}

ANÁLISIS DE SUELOS

Los análisis de suelos proporcionan una base para las recomendaciones de fertilización y enmiendas que deben aplicarse a un suelo como el yeso, en suelos alcalinos o materiales encalantes en suelos ácidos. Esta información debe interpretarse, primero en términos agronómicos y biológicos con respecto al crecimiento de las plantas, luego se interpretará la información desde un punto de vista económico a fin de determinar el nivel de retorno deseado.

\section{EL ESTIÉRCOL}

Los estiércoles y/o enmiendas orgánicas mejoran las condiciones físicas de los suelos especialmente en las regiones áridas de la costa peruana. Así mismo, la escasez del agua limita el balance apropiado con el aire del suelo, donde el estiércol contribuye notablemente a este balance (Davelouis, 1993). Cuando los suelos son irrigados los residuos orgánicos llenan los poros no capilares de los suelos arenosos y los transforman en capilares, incrementan la retentividad del agua y los convierten en suelos económicamente productivos.

El estiércol se utiliza en dosis importantes, ya que un estercolado medio supone la aplicación de $30 \mathrm{t} / \mathrm{ha}$, pero se utilizan a menudo dosis mayores a 40-50 tha. Asi mismo, no debe depreciarse su papel como suministrador de nutrientes. Por ejemplo, 30 toneladas de estiércol aportan 120, 75 y $165 \mathrm{~kg} /$ ha de $\mathrm{N}$, $\mathrm{P}_{2} \mathrm{O}_{5}$ y $\mathrm{K}_{2} \mathrm{O}$ respectivamente; además, estos nutrientes se encuentran en forma de complejos orgánicos, los cuales tienen que ser mineralizados para pasar a su forma asimilable, 
por ello no todos serán aprovechados por el primer cultivo instalado después de su aplicación (Gross, 1992; Kononova, 1982).

La única manera de contrarrestar las enormes pérdidas de la fertilidad en un suelo consiste en desarrollar sistemas apropiados de explotación y/o rotaciones de cultivos y ampliar el uso de fertilizantes orgánicos y minerales (Fassbender, 1987). Los abonos orgánicos pueden ser en primer lugar una fuente importante de microelementos, aunque el contenido de ellos en los estiércoles es muy variable y está en función del tipo de animal, alimentación, naturaleza de las camas, estado de descomposición y condiciones de almacenamiento (Loue, 1988).

\section{Relación del estiércol con la productividad}

Valverde et al. (1994), en un suelo de textura franca encontró que los niveles crecientes de estiércol de vacuno incrementaron el rendimiento de papa; pero, el fertilizante químico, solo o en mezcla con el estiércol, produjo los rendimientos más altos.

Alaluna (1995), evaluó el efecto del estiércol y el abastecimiento de los tres principales elementos ( $\mathrm{N}, \mathrm{P}$ y K ) en un suelo arenoso mediante el uso de la técnica del elemento faltante y encontró un incremento de 31,121 y $21 \%$ para el $\mathrm{N}, \mathrm{P}$ y $\mathrm{K}$ respectivamente, concluyendo en que el fósforo posiblemente sea absorbido en forma orgánica por parte del cultivo de la papa.

\section{La fertilización foliar}

La ventaja de la nutrición foliar es que proporciona un mejoramiento inmediato y es mucho más efectiva que la fertilización al suelo. En algunos casos es mucho más económica, ya que solamente se requieren cantidades pequeñas de nutrientes, los cuales se puede combinar con el programa de aplicación de productos agroquímicos. La desventaja de la nutrición foliar es que no produce un efecto residual substancial y requiere aplicarse en cada situación (Wittwell, 1964).

Bertran (1965), señala que así como ocurre con la piel de los animales, la cutícula de los vegetales goza de propiedades absorbentes. Esta característica ha sido aprovechada en agricultura para efectuar abonaduras complementarias de acción rápida. Los elementos mayores $\mathrm{N}, \mathrm{P}$ y K, lo mismo que el azufre, apenas absorbido se reparten dentro de la planta, mientras que el calcio, el magnesio, el hierro tienen tendencia a acumularse en las hojas pudiendo ser lavados luego por las aguas de lluvia.

\section{Absorción de nutrientes}

Si un cultivo estuviese sembrado en un suelo calcáreo donde los elementos menores se encuentran mayormente en la forma oxidada, es decir, en formas menos disponibles, los tejidos tendrán menor concentración de los elementos minerales ( $\mathrm{Fe}, \mathrm{Mn}, \mathrm{Zn}, \mathrm{Cu}$ y B) y las extracciones por unidad de producción serán menores sin que esto necesariamente signifique deficiencia que limite la producción (Villagarcía, 1990). En el caso de la extracción de bases cambiables se ha observado que un cultivo de papa, en la Molina, con ún rendimiento de 41 toneladas de tubérculo/ha extrae un promedio de 100,20 y 15 de $\mathrm{K}_{2} \mathrm{O}$, $\mathrm{CaO}$ y $\mathrm{MgO} \mathrm{kg} / \mathrm{ha}$ respectivamente (Ezeta, 1970).

Sumar, K. et al. (1983), en el Centro de Investigación de Cultivos Andinos de la UNSAAC (Cuzco), probaron en el cultivo de kiwicha dos niveles de fertilización mineral al $40-40-0$ y $80-80-0$ en $\mathrm{kg} / \mathrm{ha}$ y 10,20 y 30 en $\mathrm{t}$ ha de estiércol de vacuno y un testigo (0-0-0).

Se encontró que la regresión en las enmiendas orgánicas presentó una pendiente de 0,38 tha de incremento en grano por cada 10 toneladas de estiércol aplicado. Ese notable 
efecto puede deberse a la mayor conservación de humedad de la zona radicular, al incremento de temperatura durante la germinación y a la mayor capacidad de cambio del suelo.

\section{OBJETIVOS}

1 Evaluar el efecto de la fertilización al suelo, niveles de fertilización foliar y estiércol en el rendimiento y absorción total de nutrientes en la secuencia de papa y kiwicha 2.

2 Evaluar la interacción de la fertilización al suelo por niveles de fertilización foliar y estiércol en el rendimiento y absorción total de nutrientes en la secuencia de papa y kiwicha.

\section{MATERIAL Y MÉTODOS}

\section{UBICACIÓN}

El experimento se realizó en el invernadero del Laboratorio de Fertilidad de Suelos de la UNALM, sobre $238 \mathrm{msnm}$. Los datos climatológicos durante el establecimiento de los cultivos fueron: temperatura promedio mensual $17,75^{\circ} \mathrm{C}$, precipitación pluvial mensual $0,75 \mathrm{~mm}$; las características del suelo utilizado fueron de textura arena, $\mathrm{CE}: 0,51 \mathrm{dS} /$ $\mathrm{m}, \mathrm{pH}: 8,5, \mathrm{M}, \mathrm{O}: 0,38 \%$, P-disponible 5,6 ppm, $\mathrm{K}$-disponible $349 \mathrm{~kg} \mathrm{~K} \mathrm{O} / \mathrm{ha}$. De acuerdo a los resultados del análisis de suelo se trata de un suelo sin problemas de sales y de $\mathrm{pH}$, ligeramente alcalino con un bajo porcentaje de materia orgánica, con nivel bajo de fósforo y medio de potasio disponible. Los factores en estudio son nueve fórmulas de fertilización al suelo (se evaluaron los elementos; N, P, K, $\mathrm{Mg}, \mathrm{Fe}, \mathrm{Mn}$ y $\mathrm{Zn}$ más un tratamiento testigo y completo, mediante la técnica del elemento faltante), dos niveles de fertilización foliar ( $\sin$ y con) y dos de estiércol ( 0 y 25 t/ha). Los niveles de fertilización mineral al suelo para cada elemento fueron de $200 \mathrm{mg} \mathrm{kg}^{-1}\left(\mathrm{~N}, \mathrm{P}_{2} \mathrm{O}_{5}\right.$ y $\mathrm{K}_{2} \mathrm{O}$ ), $50 \mathrm{mg} \mathrm{kg}^{-1}$ ( $\mathrm{MgO}$ y Fe) y $25 \mathrm{mg} \mathrm{kg}^{-1}$
(Mn y $\mathrm{Zn}$ ). Los fertilizantes utilizados fueron urea $(45 \% \mathrm{~N})$, fosfo-humus $\left(20 \% \mathrm{P}_{2} \mathrm{O}_{5}\right)$, cloruro de potasio $\left(60 \% \mathrm{~K}_{2} \mathrm{O}\right)$, sulfato de magnesio $(9 \% \mathrm{Mg}$ ), sulfato de hierro $(19 \%$ $\mathrm{Fe})$, sulfato de manganeso ( $26 \% \mathrm{Mn}$ ), sulfato de $\mathrm{Zn}(23 \% \mathrm{Zn})$, y como fuente de fertilizante foliar se utilizó los fertilizantes de la línea ALFA.

Para evaluar el presente experimento se usó el Diseño Completo al Azar con arreglo factorial, haciendo un total de 36 tratamientos con 3 repeticiones, y analizados con la prueba estadística de Duncan, donde cada unidad experimental está constituida por una maceta de $4 \mathrm{~kg}$ de suelo. Se sembró una yema de tubérculo de papa cv. Costanera el día 05 de mayo de 1994. La fertilización foliar se fraccionó en dos aplicaciones efectuándose los días 28 de mayo y el 08 de junio respectivamente.

La cosecha se realizó los días 09 y 10 de julio, y se cosechó por separado la parte aérea (tallos y hojas), tubérculos frescos y las raíces fibrosas, para luego someterlos a las evaluaciones respectivas; una vez cosechado el experimento de la papa con la finalidad de aprovechar el efecto residual de las fuentes adicionales se sembró el cultivo de kiwicha cv. Oscar Blanco de manera directa el día 29 de agosto y se adicionó $50 \mathrm{ppm} \mathrm{N} /$ maceta a los tratamientos que tenían fertilización nitrogenada al suelo y se mantuvo la misma dosis de fertilización foliar del cultivo anterior. La cosecha de la kiwicha se efectuó los días 19 y 20 de noviembre, de manera similar a la de la papa, donde la parte aérea incluyó los tallos, hojas y panojas. Las muestras fueron lavadas en principio con agua de caño, luego con agua destilada. Esto tanto para la papa como para la kiwicha; una vez eliminada la humedad se procedió a la evaluación de peso fresco de tubérculo, para luego ponerlos en la estufa a una temperatura de $70^{\circ} \mathrm{C}$ hasta obtener peso constante y proceder a evaluar el peso de la materia seca total tanto de la 
papa como de la kiwicha. Posteriormente se procedió a la molienda de los tejidos evaluados y luego se calcinaron en una mufla a $500^{\circ} \mathrm{C}$; en ello se analizó el contenido de la absorción de elementos mediante el método de Espectrofotometría de Absorción atómica, excepto el nitrógeno que fue analizado por el método del micro Kjeldahl.

\section{RESULTADOS Y DISCUSIÓN}

Efecto primario de la fertilización mineral en el rendimientopromedio de materia seca total (mst) en la secuencia papa-kiwicha. Con la incorporación de 25 t/ha de estiércol es posible suplir los requerimientos nutricionales de los elementos Fe, Mn y Zn para el cultivo de la papa; así mismo, mediante este procedimiento se puede suplir parcialmente los requerimientos de $\mathrm{K}$ y $\mathrm{Mg}$ y de manera limitada los de $\mathrm{P}$ y N. En la kiwicha el tratamiento completo mostró ligera depresión en su rendimiento, comparado con las ausencias de $\mathrm{Mg}, \mathrm{K}$, Mn y $\mathrm{P}$ ya que estos elementos fueron favorecidos por la mineralización del estiércol adicionado al cultivo anterior.

\section{Efecto de la interacción fertilización mine- ral por niveles de fertilización foliar en el rendimiento promedio de materia seca total}

El resultado obtenido en la papa indica que los tratamientos $-\mathrm{Mn}+\mathrm{FF}$, Completo $+\mathrm{FF}$, Testigo+FF y $-\mathrm{Fe}+\mathrm{FF}$ mostraron efecto positivo a la aplicación de la fertilización foliar los cuales incrementaron lą producción en el orden de 22,$80 ; 10,62 ; 17,25$ y $3,03 \%$ con relación a los tratamientos sin fertilización foliar en cada uno de los casos, de los cuales sólo la ausencia del $\mathrm{Mn}$ permitió un incremento significativo de la producción. Sin embargo, frente a la carencia de $\mathrm{P}, \mathrm{K}, \mathrm{Mg}, \mathrm{Zn}$ y $\mathrm{N}$, la fertilización foliar actuó negativamente puesto que redujo la producción en 36,65 ; 17,$08 ; 9,57 ; 8,98$ y $6,12 \%$ respectivamente.
Este resultado confirma que la ausencia de los macroelementos primarios en el suelo genera un desbalance nutricional; en consecuencia los rendimientos más altos se consiguen con niveles altos de NPK más fertilización foliar. En la kiwicha los tratamientos con ausencia de $\mathrm{K}, \mathrm{Fe}, \mathrm{P}$, Testigo y $\mathrm{Zn}$ incrementaron la producción en 12,$60 ; 11,91 ; 9,58 ; 3,38$ y $1,60 \%$ debido a la fertilización foliar. El análisis del suelo indicó que este suelo tiene un contenido medio de potasio, el cual no fue aprovechado por el cultivo anterior y quedó un remanente para el siguiente cultivo. Las ausencias de Fe y Mn fueron suplidas mediante la fertilización foliar, ya que ellos no limitan la producción de manera significativa. La fertilización foliar frente a la falta de $\mathrm{N}$ y $\mathrm{Mg}$ en el suelo redujo significativamente la producción en 10,51 y $10,41 \%$ respectivamente.

\section{Efecto de la interacción fertilización mine- ral por niveles de estiércol en el rendimiento promedio de materia seca total}

La incorporación del estiércol incrementó la producción en 103,$19 ; 91,20 ; 61,74 ; 51,27$; 33,23 y $21,04 \%$ en ausencia de los elementos $\mathrm{N}, \mathrm{Mn}$, Testigo, P, K y Zn. El abastecimiento del nitrógeno por parte del estiércol fue positivo a pesar de que el análisis del estiércol muestra un contenido de $1,50 \%$ de $\mathrm{N}$ total, lo que hace suponer que la descomposición del mismo ha sido rápida.

La absorción del Fe inorgánico está ligada a la capacidad que tienen las raíces de reducir el $\mathrm{pH}$ y por ende el $\mathrm{Fe}^{+3}$ a $\mathrm{Fe}^{+2}$ en la rizósfera (Brown et al, 1961); así mismo este efecto fue favorecido con la incorporación del estiércol. La adición del estiércol en ausencia de $\mathrm{Mg}$ redujo la producción en $22,77 \%$, el cual se debió principalmente al antagonismo existente entre este elemento y el $\mathrm{Ca}, \mathrm{K} \mathrm{y}$ Mn provenientes del estiércol.

En la kiwicha la adición del estiércol en ausencia del $\mathrm{N}$, testigo, P y $\mathrm{K}$ favoreció 
significativamente la producción incrementando el mismo en 1024,53; 948, $18 ; 42,04$ y $12,84 \%$ respectivamente. Estos resultados nos indican que la disponibilidad del N, P y K fue favorecida por la lenta mineralización del estiércol donde es posible que el fósforo sea absorbido en forma orgánica por la planta. Bajo el mismo sistema la carencia de $\mathrm{Mn}, \mathrm{Zn}, \mathrm{Fe} \mathrm{y}$ $\mathrm{Mg}$ con adición de estiércol disminuyó la producción en 16,$51 ; 14,32 ; 11,44$ y $7,53 \%$. La ausencia del Mg mantiene la misma tendencia de producción del cultivo anterior, debido al antagonismo existente entre el $\mathrm{Mg}$ y otros cationes como el K+ $\mathrm{Ca}^{+2}, \mathrm{NH}^{+}, \mathrm{Fe}^{+2}$ y $\mathrm{Zn}^{+2}$ procedentes del suelo y de la mineralización de la materia orgánica. La disminución en ausencia de $\mathrm{Mn}, \mathrm{Fe}$, y $\mathrm{Zn}$ se debe a que estos elementos pasan a formar complejos órgano-metálicos, donde el elemento está insertado en una molécula quelatante recubierta (Heller, 1977).

\section{Efecto de la interacción fertilización mine- ral por niveles de fertilización foliar y estiércol en el rendimiento de materia seca total}

En la tabla 1, los tratamientos $-\mathrm{N}+\mathrm{FF}+\mathrm{Est}$ y $-\mathrm{N}-\mathrm{FF}+$ Est incrementan la producción de papa en 79,7 y $66,36 \%$ con relación al $-\mathrm{N}-$ FF-Est, en el cual la aplicación del estiércol favoreció la disponibilidad del $\mathrm{N}$ a nivel del suelo evitando el desbalance nutricional por la falta de este elemento; así mismo la fertilización foliar no mostró un efecto significativo en ia producción. En la kiwicha como se puede apreciar en el cuadro 1 , el tratamiento $-\mathrm{N}-$ $\mathrm{FF}+$ Est muestra él más alto rendimiento seguido por $-\mathrm{N}+\mathrm{FF}+\mathrm{Est}$, los cuales incrementan la producción en 2081,66 y $1690,00 \%$ con relación al $-\mathrm{N}-\mathrm{FF}-$ Est. Este resultado indica que el estiércol aporta más nitrógeno en el segundo cultivo debido al lento proceso de descomposición.

El tratamiento -P-FF+Est incrementó la producción de materia seca de papa en $80,46 \%$ con relación al-P-FF-Est; sin embargo el
$-\mathrm{P}+\mathrm{FF}-$ Est $\mathrm{y}-\mathrm{P}+\mathrm{FF}+$ Est tuvieron efectos negativos en la producción, con lo cual queda demostrado que el $P$ genera más desequilibrio que el $\mathrm{N}$ a nivel del suelo.

El tratamiento $-\mathrm{K}-\mathrm{FF}+\mathrm{Est}$ superó significativamente la producción de papa con relación a los tratamientos $-\mathrm{K}+\mathrm{FF}-\mathrm{Est},-\mathrm{K}+\mathrm{FF}+\mathrm{Est}$ y-K-FF-Est; este resultado indica que la fertilización foliar limita la producción en presencia del estiércol. En el efecto residual dicho efecto se revierte teniendo el rendimiento más alto con el tratamiento $-\mathrm{K}+\mathrm{FF}+$ Est.

El tratamiento $-\mathrm{Mg}+\mathrm{FF}+$ Est limitó de manera significativa la producción en la secuencia papa-kiwicha, debido a que este elemento tiene gran movilidad en el suelo y su absorción por parte de los cultivos es principalmente por flujo de masas después del nitrógeno y calcio, donde estos últimos elementos tienen cierta ventaja debido justamente a la adición del nitrógeno mediante la fertilización química y porque se trata de un suelo con $\mathrm{pH}$ alcalino donde hay predominancia de calcio en el medio. También cabe mencionar que esta deficiencia no puede ser corregida por la fertilización foliar porque el agua de riego utilizada tiene un alto contenido de calcio y sodio. La interacción fertilización foliar por estiércol no tuvo efecto significativo en ausencia de Fe y Zn pero sí en Mn para el caso de la papa. En la kiwicha se obtuvo una reducción significativa en ausencia de Mn y Zn pero no en ausencia de Fe. La respuesta negativa en el segundo cultivo se debe a que el estiércol se encuentra en una etapa de humificación muy avanzada que favorece la quelación de estos elementos y limita su disponibilidad.

\section{Absorción total de nutrientes en la secuen- cia papa-kiwicha}

La incorporación del estiércol en la papa favoreció de manera significativa la absorción del N, P, K y Mg. Sin embargo la absorción del Fe, Mn y Zn fue favorecida por la fertilización foliar. Así mismo la ausencia de los ele- 
Efecto de la interacción de la fertilización mineral, niveles de fertilización foliar y estiércol en el rendimiento promedio de materia seca total en la secuencia papa-kiwicha.

\begin{tabular}{|c|c|c|c|c|c|}
\hline \multicolumn{3}{|c|}{ Tratamiento } & \multirow{2}{*}{$\begin{array}{l}N:^{2} \\
\text { Repet }\end{array}$} & \multicolumn{2}{|c|}{$\begin{array}{l}\text { Rendimiento de Materia Seca Total } \\
\qquad(g / \text { maceta })\end{array}$} \\
\hline $\begin{array}{l}\text { Fertilización } \\
\text { al Suelo }\end{array}$ & $\begin{array}{l}\text { Fertilización } \\
\text { Foliar }\end{array}$ & Estiércol & & Papa & Kiwicha \\
\hline \multirow{4}{*}{$N$} & - & - & 03 & $04,44 \mathrm{NM}$ & $00,60 \mathrm{~N}$ \\
\hline & . & + & 03 & 07,32 GHIJ & $13,09 \mathrm{M}$ \\
\hline & + & - & 03 & $03,08 \mathrm{~N}$ & $01,51 \mathrm{~N}$ \\
\hline & + & + & 03 & 07,97 DEFGHI & $10,74 \mathrm{M}$ \\
\hline \multirow{4}{*}{ F } & $\cdot$ & - & 03 & $04,30 \mathrm{MN}$ & $13,00 \mathrm{M}$ \\
\hline & - & + & 03 & 07,76 EFGHJ & 21,67 CDEFGHI \\
\hline & + & - & 03 & $03,54 \mathrm{~N}$ & $17,01 \mathrm{~L}$ \\
\hline & + & + & 03 & $04,10 \mathrm{MN}$ & 20,97 FGHIJ \\
\hline \multirow{4}{*}{$\mathrm{K}$} & - & $\cdot$ & 03 & $05,79 \mathrm{JKLM}$ & 20,33 HIJK \\
\hline & . & + & 03 & $10,25 B C$ & 21,75 CDEFGHI \\
\hline & + & - & 03 & 06,78 HIJK & 21,73 CDEFGHI \\
\hline & + & + & 03 & $06,51 \mathrm{JKL}$ & $25,64 \mathrm{~A}$ \\
\hline \multirow{4}{*}{$\mathrm{Mg}$} & - & & 03 & $07,41 \mathrm{GHIJ}$ & 23,43 ABCDEFG \\
\hline & - & + & 03 & 07,63 FGHIJ & 24,37 ABC \\
\hline & + & - & 03 & 08,75 CDEFGH & $\begin{array}{l}23,73 \text { ABCDEF } \\
1909 \mathrm{KI}\end{array}$ \\
\hline & + & + & 03 & $04,85 \mathrm{KLMN}$ & \\
\hline \multirow{4}{*}{$\mathrm{Fe}$} & - & - & 03 & $07,43 \mathrm{GHU}$ & 21,29 DEFGHL \\
\hline & - & + & 03 & $09,73 \mathrm{BCDE}$ & $18,85 \mathrm{JKL}$ \\
\hline & $\div$ & - & 03 & 09,73 CDEFGH & $23,82 \mathrm{ABCDE}$ \\
\hline & + & + & 03 & 08,96 CDEFG & 21,10 EFGHI \\
\hline \multirow{4}{*}{$\mathrm{Mn}$} & - & - & 03 & $03,10 \mathrm{~N}$ & $25.11 \mathrm{AB}$ \\
\hline & - & + & 03 & $11,40 \mathrm{AB}$ & $19,34 \mathrm{JKKL}$ \\
\hline & + & . & 03 & 07,98 DEFGHI & $23,34 \mathrm{BCDEFG}$ \\
\hline & + & + & 03 & $09,89 \mathrm{BCD}$ & 21,12 EFGHJ \\
\hline \multirow{4}{*}{$\mathrm{Zn}$} & - & * & 03 & $06,52 \mathrm{WKL}$ & 20,66 GHIJ \\
\hline & - & + & 03 & $12,17 \mathrm{~A}$ & $20,49 \mathrm{HIJ}$ \\
\hline & + & - & 03 & $09,63 \mathrm{BCDEF}$ & $24,03 \mathrm{ABCD}$ \\
\hline & + & + & 03 & $07,38 \mathrm{GHJ}$ & $17,78 \mathrm{KL}$ \\
\hline \multirow{4}{*}{ Completo } & $=$ & - & 03 & 08,32 COEFGHI & 22,77 BCDEFGH \\
\hline & - & + & 03 & $09,76 \mathrm{BCDE}$ & $20,44 \mathrm{HJ}$ \\
\hline & + & . & 03 & $09,90 \mathrm{BCD}$ & $22,53 \mathrm{BCOEFGH}$ \\
\hline & + & + & 03 & $10,10 \mathrm{BC}$ & $17,38 \mathrm{~L}$ \\
\hline \multirow{4}{*}{ Testigo } & - & $\cdot$ & 03 & $04,22 \mathrm{MN}$ & $\infty 0,92 \mathrm{~N}$ \\
\hline & - & + & 03 & $06,55 \mathrm{IJKL}$ & $11,50 \mathrm{M}$ \\
\hline & + & . & 03 & 04,72 LMN & $01,28 \mathrm{~N}$ \\
\hline & + & + & 03 & 07,91 DEFGHIJ & $11,56 \mathrm{M}$ \\
\hline \multirow{4}{*}{ Promedio } & - & - & 27 & $05,73 \mathrm{C}$ & $16,46 \mathrm{C}$ \\
\hline & - & + & 27 & $09,17 \mathrm{~A}$ & $19,06 \mathrm{~A}$ \\
\hline & + & " & 27 & $07,01 \mathrm{~B}$ & $17,67 \mathrm{~B}$ \\
\hline & + & + & 27 & 07.528 & $18,38 \mathrm{AB}$ \\
\hline$C . V \cdot(\%)$ & & & & 14,37 & 08,05 \\
\hline DLS 0.05 & & & & 01,722 & 02,346 \\
\hline
\end{tabular}

Nota Cuando se compara dos tratamientos y se tiene al menos una letra que se repite en el análisis estadístico, esto indica que ambos tratamientos tienen un comportamiento similar; en caso contrario existen diferencias estadísticas significativas. 
mentos antagónicos también fue comprobada como es el caso de los elementos $\mathrm{Mn} / \mathrm{Mg}, \mathrm{Fe} /$ $\mathrm{Zn}, \mathrm{P} / \mathrm{Zn}$, etc., ya que la ausencia de uno de ellos favorecía la mayor absorción del otro. En la kiwicha la absorción del P y K aumentó considerablemente con la incorporación del estiércol, sin embargo tuvo efecto negativo en la absorción del $\mathrm{Mg}$, Fe, Mn y Zn. La fertilización foliar sólo tuvo efecto positivo en aquellos tratamientos con buen equilibrio nutricional a nivel del suelo.

\section{CONCLUSIONES}

1. En un suelo de irrigación con la incorporación de 25 tha de estiércol es posible suplir los requerimientos nutricionales de Fe, Mn y Zn para la papa, así mismo mediante este procedimiento se puede suplir parcialmente los requerimientos de $\mathrm{K} y$ $\mathrm{Mg}$ y de manera limitada los de P y $\mathrm{N}$.

2. La incorporación del estiércol en ausencia del N, P y K permitió incrementar la producción de papa en 103,$19 ; 51,27$ y $33,22 \%$ con relación a los tratamientos sin estiércol, y tuvo efecto depresivo en ausencia del $\mathrm{Mg}$ disminuyendo la producción en $24,27 \%$.

3. La fertilización foliar incrementó la producción en el tratamiento completo, testigo, $-\mathrm{Mn},-\mathrm{Fe}$ y $-\mathrm{Zn}$ los cuales tuvieron buen balance nutricional a nivel del suelo y demostró un efecto negativo frente a la falta de $\mathrm{N}, \mathrm{P}$ y $\mathrm{K}$.

4. La interacción del estiércol por fertilización foliar fue positiva en los tratamientos completo y testigo; de este modo la falta de cualquier elemento generó la disminución en el rendimiento de la papa como consecuencia del desbalance nutricional.

5. En la kiwicha el efecto residual del estiércol favoreció significativamente la disponibilidad de $\mathrm{N}, \mathrm{P}, \mathrm{y} \mathrm{K}$, limitando la disponibilidad de $\mathrm{Mn}, \mathrm{Mg}$, Fe y $\mathrm{Zn}$.
6. La absorción de los elementos $\mathrm{N}, \mathrm{P}, \mathrm{K}$, $\mathrm{Fe}, \mathrm{Zn}$ y $\mathrm{Mn}$ fue favorecida por el estiércol en la papa y en la kiwicha este efecto fue significativo en el caso del $\mathrm{N}$ y $\mathrm{P}$ y se limitó ligeramente la absorción del Fe, Mn, y Zn. Asî mismo la ausencia de un elemento antagónico como es el caso del fósforo permitió una mayor absorción de $\mathrm{Zn}$ en los dos cultivos.

\section{RECOMENDACIONES}

1. Para un suelo de irrigación de la costa peruana, se recomienda la incorporación de 25 tha de estiércol, el cual permitirá mejorar las propiedades del suelo y actuará como una fuente de nutrientes de $\mathrm{Fe}, \mathrm{Mn}$ y $\mathrm{Zn}$ pero a medida que aumenta su descomposición permitirá abastecer parcialmente los requerimientos de $\mathrm{P}, \mathrm{K}$ y $N$ en el orden indicado; por tanto se debe incorporar campaña tras campaña para evitar la quelación de los elementos menores.

2. En un programa de fertilización es recomendable realizar el análisis del suelo, fuente orgánica y agua de riego y así evitar los desbalances nutricionales a nivel del suelo.

3. Realizar mayor números de experimentos en suelos de diferentes texturas, estiércol y fertilización foliar, en secuencias diferentes de cultivos indicadores.

\section{LITERATURA CITADA}

Alaluna, G. E. 1995. Efecto de tres fuentes de materia orgánica en una rotación de papa-cebada en suelo de la costa central (Huaral), bajo condiciones de invernadero. Tesis de Ing. Agrónomo. UNALM. Lima.

Bertran, C. L. 1965. Nutrición en las plantas y fertilización en el Perú. S.C.P.A. Misión de los Andes. Bogotá. 
Brown, J. C. et al. 1961 Iron clororsis in soybeans as related to the genotype of rootstock III. Chlorosis susceptibility and reductive capacity at the root. Soil Science 91, 127-132.

Davelouis, J. 1993. Materia orgánica y abonos orgánicos conceptos modernos de su manejo. Copia mimeografiada. UNALM. Lima.

Ezeta, S. F. 1970. Absorción de nutrientes por el cultivo de papa (Solanum andigenum). Tesis Ing. Agrónomo. UNALM. Lima.

Fassbender, H. W. 1978. Química de suelos con énfasis en suelos de latinoamérica. Ed. IICA. San José de Costa Rica.

Gross, A. 1992. Abonos. Guía de práctica de la fertilización Ediciones Mundi Prensa. Madrid.

Heller, R. 1977. Abrege off physilogie vegetale. I. Nutriun. Masson et Cie (de) $244 \mathrm{pp}$.

Kononova, M. 1982. Materia orgánica del suelo, su naturaleza, propiedades y métodos de investigación. Barcelona.
Loue, A. 1988. Los microelementos en agricultura. Ediciones Mundi Prensa. Madrid.

Sumar, K. y Sadis, A. 1983. La kiwicha. Centro de Investigaciones de Cultivos Andinos. Programa de Investigaciones Amaranthus. UNSAAC. Cuzco.

Tisdale, S. L. and W. NELSON. 1991. Fertilidad de los suelos y fertilizantes. Ediciones UTEHA. México, D. F.

Valverde, F. 1994. Efecto de la aplicación de abonos orgánicos e inorgánicos en el rendimiento y calidad de la papa y las propiedades del suelo. FORTIPAPA. Informe Anual del Programa de Raíces y Tubérculos. Quito.

Villagarcia, H. S. et al. 1990. Resultados de ensayos de campo sobre fertilización y nutrición mineral en el cultivo de la papa. Campaña 1989-1990. UNALM-CIP. Lima.

Wittwell, S. M. 1964. Foliar absorption of plant nutrients. In Frontiers of plant Sciences. Institute for Advancement of Science. 